Full length article

\title{
Walking behavior over multiple obstacles in people with Parkinson's disease
}

\author{
Diego Orcioli-Silva ${ }^{\mathrm{a}, *}$, Fabio Augusto Barbieri ${ }^{\mathrm{b}}$, Lucas Simieli ${ }^{\mathrm{b}}$, Rodrigo Vitorio ${ }^{\mathrm{a}}$, \\ Paulo Cezar Rocha dos Santos ${ }^{a}$, Victor Spiandor Beretta ${ }^{a}$, Lilian Teresa Bucken Gobbi ${ }^{a}$ \\ a Universidade Estadual Paulista (Unesp), Instituto de Biociências, Posture and Gait Studies Laboratory (LEPLO), Rio Claro, Brazil \\ ${ }^{\mathrm{b}}$ Universidade Estadual Paulista (Unesp), Faculdade de Ciências, Human Movement Research Laboratory (MOVI-LAB), Bauru, Brazil
}

\section{A R T I C L E I N F O}

\section{Keywords:}

Multiple obstacles

Gait

Parkinson's disease

\begin{abstract}
A B S T R A C T
The presence of a second obstacle changed the planning and adjustments for obstacle avoidance performance, but this context is poorly understood in Parkinson's disease (PD). The aim of this study was to investigate the walking behavior over multiple obstacles in people with PD. Nineteen people with PD and 19 healthy individuals walked across an $8 \mathrm{~m}$ pathway, performing three trials for following conditions: unobstructed walking, walking with one obstacle avoidance (Single), and walking with two obstacles avoidance (Double). In the Double condition, the analysis was performed only for the first obstacle (First Double). The dependent variables were calculated separately for the approach and crossing phases in the obstacle conditions. The main results show that people with PD decreased single support and increased double support phase in both Single and Double conditions compared to the unobstructed walking. Both groups increased stride duration during approach phase in the Double condition compared to the unobstructed walking and Single conditions. The presence of the second obstacle led to a decrease in trailing toe clearance during obstacle avoidance of the First Double. In conclusion, people with PD use a conservative strategy while approaching obstacles. Both groups need more time to obtain and process environmental information and plan the action in environments with multiple obstacles. The smaller leading toe clearance might be an indicative that the presence of a second obstacle increase the likelihood of tripping during obstacle avoidance in both people with PD and healthy individuals.
\end{abstract}

\section{Introduction}

Obstacle avoidance during walking is a complex task for people with Parkinson's disease (PD), which increases the likelihood of tripping, being that this is one of the main causes of falls in this population [1]. Despite obstacle avoidance being a common daily activity, people with PD present deficits in cognitive domains, the sensorimotor system, planning and control of movements, acquisition of visual and somatosensory information, and integration of the systems that may impair obstacle avoidance during walking [2-5]. Due to these impairments, people with PD decrease gait automaticity $[4,6]$, presenting difficulties in adjusting foot position, identifying environmental characteristics with precision, and planning a movement appropriately in order to ensure the success of obstacle avoidance [7].

Previous studies have indicated that in the presence of a single obstacle, people with PD increase step width, spend less time in the swing phase, and reduce gait velocity and the horizontal distance between the feet and the obstacle compared to healthy individuals [8-11]. This behavior indicates that people with PD are more unstable [8] and demonstrate hypometria (reduced movement amplitude) and bradykinesia (slowness of movement) [10,11] during obstacle avoidance. However, during activities of daily living, people are often confronted with more than one obstacle in their travel path $[12,13]$, and this has not yet been investigated in people with PD. Environments with two or more obstacles are more complex and might increase the risk of tripping during obstacle avoidance in people with PD.

The presence of a second obstacle changes the planning and adjustments for obstacle avoidance [14]. Healthy individuals, in the presence of a second obstacle, decrease the crossing step velocity and landing distance for both the first and second obstacles, indicating a cautious strategy but with a higher risk of falling [13]. However, this is the first study to investigate the influence of secondary obstacle avoidance during walking in people with PD. Previous studies involving situations of stepping on one and/or two targets positioned in the pathway found that stepping accuracy of older adult fallers and people with PD declined in the presence of second target $[15,16]$. The loss of accuracy was associated with early gaze deviation from the stepping location (target), suggesting that older adult fallers and people with PD

\footnotetext{
* Corresponding author at: Universidade Estadual Paulista (Unesp), Instituto de Biociências, Posture and Gait Studies Laboratory (LEPLO), avenida 24-A, 1515, Bela Vista, 13506-900, Rio Claro, SP, Brazil.

E-mail address: diego_orcioli@hotmail.com (D. Orcioli-Silva).
} 


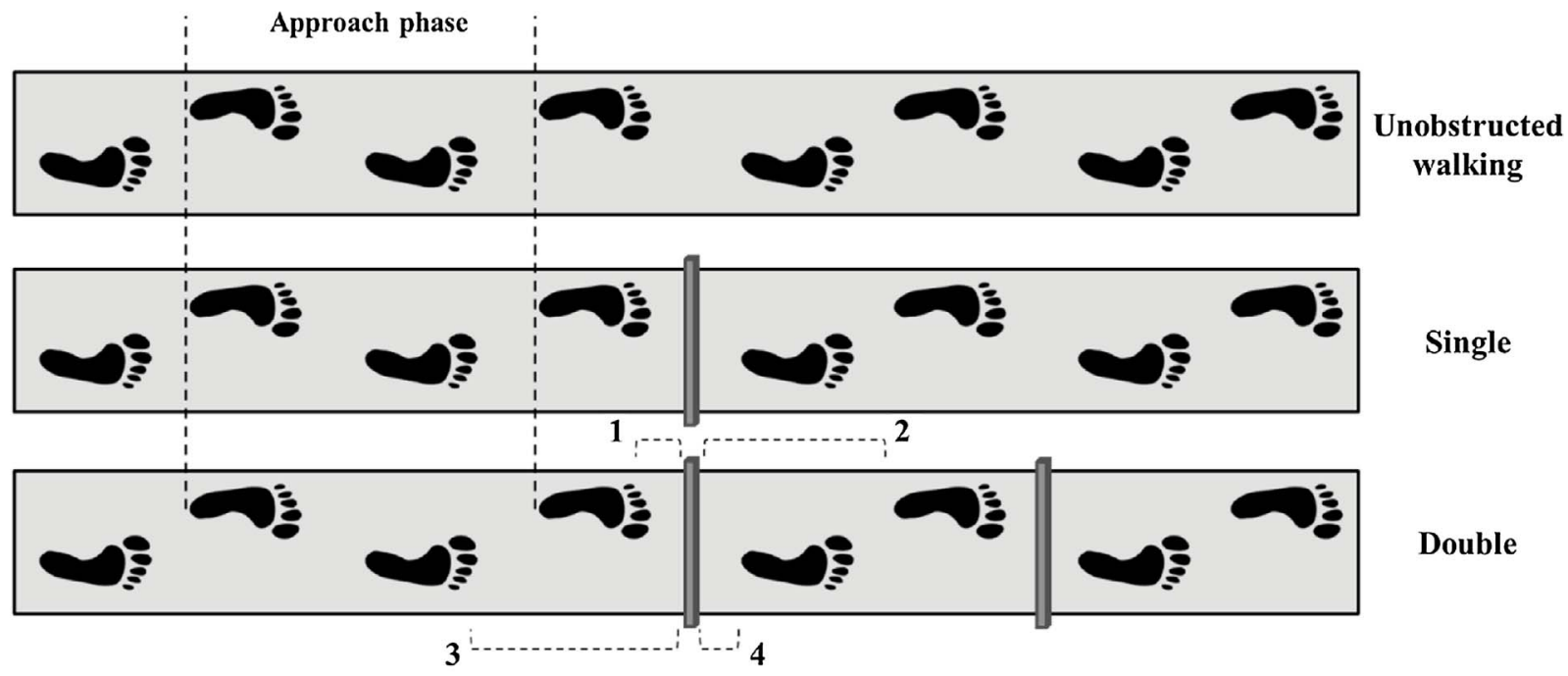

1 - Trailing horizontal distance before obstacle; 2 - Trailing horizontal distance after obstacle; 3 - Leading horizontal distance before obstacle; 4 - Leading horizontal distance after obstacle.

Fig. 1. Experimental setup and areas where gait variables were calculated.

prioritize the planning of future actions over the execution of ongoing steps $[15,16]$. As such, one might expect that people with PD, during multiple obstacles condition, look away before completing the ongoing step over the first obstacle, which would increase the risk of tripping. Environments with multiple obstacles require sequential adjustments for safe navigation and increase the demand on central processing. Individuals have to deal with an increased amount of relevant environmental information, which have to be processed and incorporated to the motor plan [17]. As people with PD present cognitive and sensorimotor deficits [2], they might be at increased risk while walking over multiple obstacles.

Therefore, the aim of the current study was to investigate the walking behavior over multiple obstacles in people with PD and to compare the behavior with healthy individuals. It was hypothesized that (a) the presence of a second obstacle in the travel path would cause gait adjustments in the approach phase (decrease in stride length and velocity and increase in stride duration) and crossing phase (decrease in step length and velocity and increase in step duration). In addition, (b) due to PD-related limitations in central processing while computing the second obstacle into motor plan, the presence of a second obstacle on the floor would magnify the obstacle avoidance deficits in people with PD.

\section{Methods}

\subsection{Participants}

After signing the informed consent, 20 individuals with PD (PD group), according to the UK Brain Bank Criteria [18], and 20 healthy matched-individuals (control group) participated in the study, which had been approved by the local Ethics Committee (\#26664014.5.0000.5465). Individuals were included if they met the following inclusion criteria: over 60 years of age, able to walk without the use of any aids, not presenting cognitive decline, and not presenting musculoskeletal, orthopedic and/or visual impairments. In addition, an inclusion criterion was that the people with PD had to be taking PD medication and be classified in stages I-III of the Hoehn \& Yahr Scale (H \& Y [19]). PD group were tested in the "on state" of regular PD medication (approximately $1 \mathrm{~h}$ after having taken a dose). The Levodopa equivalent dose was calculated according to Tomlinson's suggestions [20].

\subsection{Experimental protocol}

The PD group was clinically evaluated, by a movement disorders specialist, to determine the motor symptom severity and stage of PD, using the motor portion of the Unified Parkinson's Disease Rating Scale (UPDRS III [21]) and the H\&Y scale, respectively. Cognitive aspects were assessed using the Mini Mental State Examination (MMSE [22]) in both groups.

Participants received the instruction to walk over an $8 \mathrm{~m}$ wooden pathway at a self-selected speed. Each participant performed three trials for each condition: (i) unobstructed walking (UnW); (ii) walking with one obstacle avoidance (Single); (iii) walking with two obstacles avoidance (Double). The trials were randomized for each participant. The obstacle was positioned midway along the pathway. The starting position was adjusted for each participant to ensure comfortable obstacle crossing with the right limb. In the Double condition, the second obstacle (Second Double) was placed in the travel pathway $108 \mathrm{~cm}$ (approximate stride length of people with PD [9]) distant from the first obstacle (First Double), ensuring obstacle crossing for the Second Double with the right lower limb (Fig. 1). The obstacles were made of foam and were $15 \mathrm{~cm}$ in height, $3 \mathrm{~cm}$ thick, and $60 \mathrm{~cm}$ wide. The obstacle height and inter-obstacle distance was fixed for this study, this choice was based on the fact that many obstacles in our environment are not scaled to an individual's size or proportions [13].

Gait parameters were recorded using a GAITRite system with a frequency of 200 samples/s. For both obstacle conditions, the dependent variables were calculated separately for the approach (final stride before the obstacle) and crossing phases (Fig. 1). In the Double condition, the analysis was performed only for the First Double. For the approach phase and UnW condition (mid-pathway stride), the variables stride length, width, duration, and velocity and the time percentage in the swing, single support, and double support phases were calculated. With regard to the crossing phase, the calculated variables were step length, width, duration, and velocity and the time percentage in the swing phase. In addition, leading and trailing foot placement before the obstacle (horizontal distance from the metatarsal marker to the marker at the obstacle), leading and trailing toe clearance (vertical distance from the metatarsal marker to the marker at the obstacle at the crossing moment), and leading and trailing foot placement after obstacle crossing (horizontal distance from the calcaneus marker to the marker at the obstacle) were also analyzed. These variables were recorded using an optoelectronic tridimensional system (OPTOTRAK Certus) 
Table 1

Characteristics of the PD group and control group.

\begin{tabular}{lll}
\hline Demographic measure & PD group & Control group \\
\hline Male/female & $10 / 9$ & $10 / 9$ \\
Age (years) & $71.53 \pm 6.39$ & $70.37 \pm 6.25$ \\
Body height (cm) & $161.72 \pm 8.13$ & $161.04 \pm 7.12$ \\
Body mass (Kg) & $67.38 \pm 9.44$ & $72.82 \pm 14.86$ \\
Mini Mental State Examination (score) & $27.50 \pm 1.70$ & $28.35 \pm 1.30$ \\
UPDRS - motor portion (score) & $27.05 \pm 7.60$ & \\
Hoehn \& Yahr (stage) & $2.13 \pm 0.40$ & \\
Levodopa equivalent dose (mg/day) & $585.23 \pm 389.56$ & \\
&
\end{tabular}

with a frequency of 100 samples/s, positioned in the right sagittal plane. Four active markers were fixed at the following anatomic points: a) 5th metatarsal and lateral face of the calcaneus of the right foot; b) 1st metatarsal and medial face of the calcaneus of the left foot. Additionally, one marker was fixed at the top edge of the obstacle. Marker trajectories were filtered with a fifth-order Butterworth low-pass filter, with a cutoff frequency of $6 \mathrm{~Hz}$. The variables were calculated using an algorithm created in Matlab 7.0 (The Maths Works Inc.).

\subsection{Data analysis}

Statistical analysis was performed using SPSS 22.0 for Windows . Significance was set at $\mathrm{p} \leq 0.05$. T-tests were performed to compare groups for demographic data. The statistical analysis was performed separately for the approach and crossing phases. Gait parameters of the approach phase were analyzed by two-way analysis of variance (ANOVA), with group (PD group $\times$ control group) and condition (UnW $\times$ Single $\times$ Double) as factors, with repeated measures for the second factor. Due the single distance between obstacles used in the Double condition, analysis of covariance (ANCOVA), with step length during UnW as covariate, was used to compare the kinematic variables in the crossing phase. In addition, the crossing phase variables that are not influenced by a single distance between obstacle (horizontal distance before obstacle and toe clearance) were analyzed by two-way ANOVA. The Bonferroni post hoc test was used to localize the differences when ANOVA revealed significant interactions. The partial eta squared $\left(\mathrm{p \eta}^{2}\right)$ statistic provided estimates of the effect sizes.

\section{Results}

The characteristics of the PD group and control group are presented in Table 1. One individual with PD was not able to perform the obstacle avoidance in the Double condition and was excluded together with their pair (individual of the control group) from the analysis.

\subsection{Approach phase}

Interaction between group and condition was identified for stride length $\left(\mathrm{F}_{2,72}=5.895, \mathrm{p}=0.004, \mathrm{p}^{2}=0.141\right)$, single support phase $\left(\mathrm{F}_{2,72}=4.508, \mathrm{p}=0.014, \mathrm{p \eta}^{2}=0.111\right)$, and double support phase $\left(\mathrm{F}_{2,72}=3.029, \mathrm{p}=0.055, \mathrm{p \eta}^{2}=0.078\right.$ - trend) (Fig. 2). Post hoc analysis indicated that the PD group, in all conditions, presented shorter stride length (UnW: $p=0.028$, Single: $p=0.002$; Double: $\mathrm{p}=0.002$ ) and single support phase (UnW: $\mathrm{p}=0.013$, Single: $\mathrm{p}=0.001$, Double: $\mathrm{p}=0.003$ ), and greater double support phase (UnW: $p=0.035$, Single: $p=0.009$, Double: $p=0.02$ ) compared to the control group. In addition, both groups decreased stride length in the Single ( $\mathrm{p}<0.001, \mathrm{p}=0.005$, respectively) and Double conditions ( $\mathrm{p}<0.001, \mathrm{p}=0.018$, respectively) in comparison to the UnW condition, being that the PD group presented an average reduction of $9.76 \%$, while the average reduction in the control group was $3.64 \%$. Finally, the PD group decreased the single support phase and increased the double support phase in the Single $(\mathrm{p}<0.001, \mathrm{p}=0.002$, respectively) and Double conditions $(\mathrm{p}<0.001, \mathrm{p}=0.005$, respectively) compared to the UnW condition, while the control group did not present changes in these variables.

ANOVA also indicated a main effect of group for stride velocity $\left(\mathrm{F}_{1,36}=7.235, \mathrm{p}=0.011, \mathrm{p}^{2}=0.167\right)$ and a main effect of condition for stride duration $\left(\mathrm{F}_{2,72}=5.445 ; \mathrm{p}=0.006, \mathrm{p \eta}^{2}=0.131\right)$, stride velocity $\left(\mathrm{F}_{2,72}=58.514, \mathrm{p}<0.001, \mathrm{p \eta}^{2}=0.619\right)$, and swing phase percentage $\left(\mathrm{F}_{2,72}=8.63, \mathrm{p}<0.001, \mathrm{p}^{2}=0.193\right)$ (Table 2). The PD group presented shorter stride length and single support phase greater double support phase and slower stride velocity compared to the control group. In the Single and Double conditions, participants showed slower stride velocity ( $\mathrm{p}<0.001, \mathrm{p}<0.001$, respectively), and greater swing phase ( $\mathrm{p}=0.002, \mathrm{p}=0.006$, respectively) compared to the UnW condition. In addition, the participants increased stride duration in the Double condition compared to the UnW condition $(\mathrm{p}=0.019)$ and Single condition $(\mathrm{p}=0.034)$.

\subsection{Crossing phase}

ANCOVA revealed a main effect of condition for step length $\left(\mathrm{F}_{1,35}=27.738, \quad \mathrm{p}<0.001, \quad \mathrm{p}^{2}=0.442\right), \quad$ step velocity $\left(\mathrm{F}_{1,35}=13.117, \mathrm{p}=0.001, \mathrm{p \eta}^{2}=0.273\right)$ and foot placement after obstacle crossing $\left(\mathrm{F}_{1,35}=4.614, \mathrm{p}=0.039, \mathrm{p}^{2}=0.116\right)$ for leading limb. Both PD and control group presented shorter step length, horizontal distance after obstacle and slower step velocity in the Double condition compared to Single condition. With regard to the trailing limb, a main effect of condition was indicated for step length $\left(\mathrm{F}_{1,35}=59.492, \mathrm{p}<0.001, \mathrm{p} \eta^{2}=0.63\right)$, swing phase $\left(\mathrm{F}_{1,35}=8.186\right.$, $\left.\mathrm{p}=0.007, \mathrm{p \eta}^{2}=0.190\right)$, step velocity $\left(\mathrm{F}_{1,35}=44.878, \mathrm{p}<0.001\right.$, $\left.\mathrm{p \eta}^{2}=0.652\right)$ and foot placement after obstacle crossing $\left(\mathrm{F}_{1,35}=50.946, \mathrm{p}<0.001, \mathrm{p}^{2}=0.593\right)$. In the Double condition, participants showed shorter step length, swing phase, horizontal distance after obstacle and slower step velocity than the Single condition. There was no main effect of group or interaction between group and condition (Table 3).

ANOVA also revealed a main effect of group for leading foot placement before the obstacle $\left(\mathrm{F}_{1,36}=7.604, \mathrm{p}=0.009, \mathrm{p} \eta^{2}=0.174\right)$ and leading toe clearance $\left(\mathrm{F}_{1,36}=4.891, \mathrm{p}=0.033, \mathrm{p}^{2}=0.120\right)$. PD group presented shorter horizontal distance before obstacle and lower toe clearance compared to control group. In addition, a main effect of condition was found for trailing toe clearance $\left(F_{1,36}=13.170\right.$, $\mathrm{p}=0.001, \mathrm{p \eta}^{2}=0.268$ ). Participants reduced the toe clearance in Double condition compared to Single condition. There was no interaction between group and condition (Table 3).

\section{Discussion}

The aim of the current study was to investigate the walking behavior over multiple obstacles of people with $\mathrm{PD}$ and to compare the behavior with healthy individuals. The main finding of this study show that one or two obstacles on the floor have similar influence on the gait of people with $\mathrm{PD}$. Both groups, in the approach phase, decreased stride length in the obstacle conditions compared to the UnW, being that the PD group presented a more pronounced decrease in the stride length $(9.76 \%)$. In addition, PD group increased double support phase and decreased single support phase during the approach phase, regardless of the number of obstacles. Both groups increased stride duration during more complex tasks (Double condition) in the approach phase. The presence of a second obstacle required adjustments in crossing parameters of the First Double, mainly in a decrease in toe clearance. In addition, the PD group presented smaller leading horizontal distance before the obstacle and toe clearance. Based on our results, the following paragraphs discuss arguments for the complexity of the Double condition and adjustments performed by participants, especially for people with $\mathrm{PD}$, and present interpretations of the effects of obstacle avoidance in people with PD. 

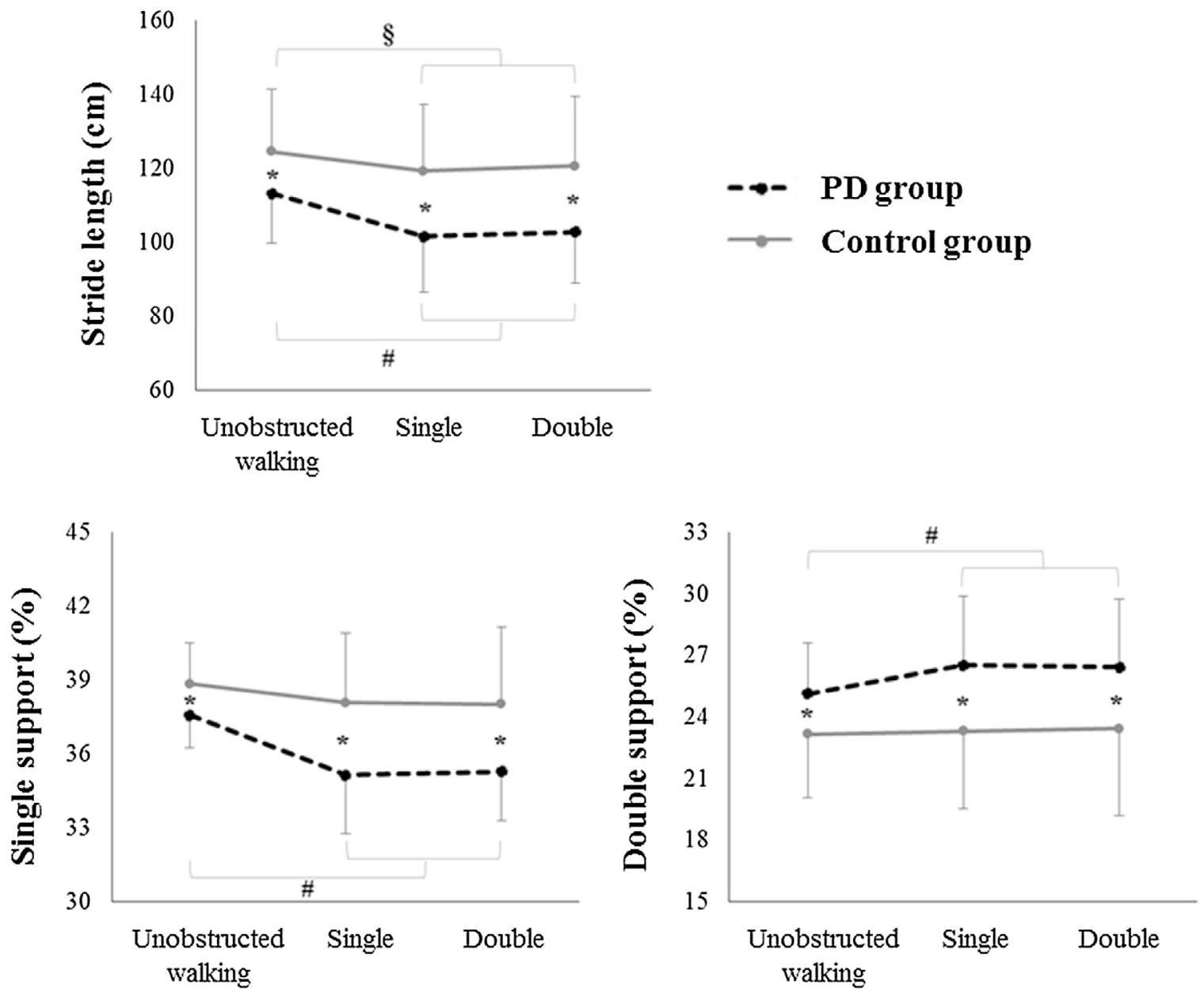

Fig. 2. Interactions between groups and conditions for approach phase.

To deal with the increased sensorimotor and central processing demands of the task (environment with obstacles), people with PD adopted a more conservative locomotor behavior during approach phase than healthy individuals, increasing the stability regardless of the number of obstacles. Besides showing a greater reduction of the stride length, the people with PD modulate the single support and double support phases. The single support phase is considered the most unstable phase of gait, due to only one foot touching the ground; in contrast, double support is the most stable phase, because both feet touch the ground [23]. An increased double support phase and decreased single support phase may relate to participants choosing to shorten the unstable phases of the gait cycle during a complex task. This is even more pronounced in environments with multiple obstacles, where people with PD decrease stride duration. Previous research has demonstrated that a decrease in stride time facilitates balance control in the medio-lateral and fore-aft directions [24]. Taken together, these results showed that people with PD use conservative strategy during the obstacle approach to compensate for postural instability [8].

Unexpectedly, both the PD and control groups increased stride duration in the approach phase during the Double condition. Environments with multiple obstacles increase motor, attentional, and sensory demands, suggesting that both groups need more time to obtain environmental information, process information, and plan the action. The planned and executed adjustments during the approach phase are necessary to ensure success in the obstacle crossing action [25]. This was confirmed by the greater adjustments in the obstacle crossing of the First Double compared to Single condition. Obstacle crossing planning requires recruitment of the frontoparietal networks [26,27], which can

Table 2

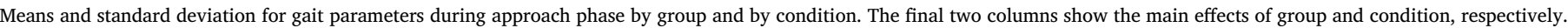
UnW: Unobstructed walking.

\begin{tabular}{|c|c|c|c|c|c|c|}
\hline Gait parameters & Group & Unobstructed walking & Single & Double & Effects of group & Effects of condition \\
\hline \multirow[t]{2}{*}{ Stride width $(\mathrm{cm})$} & PD & $12.25 \pm 2.71$ & $11.77 \pm 2.56$ & $12.56 \pm 2.66$ & \multirow[t]{2}{*}{$\mathrm{ns}$} & \multirow[t]{2}{*}{ ns } \\
\hline & Control & $10.90 \pm 2.33$ & $10.53 \pm 3.11$ & $10.73 \pm 2.93$ & & \\
\hline \multirow[t]{2}{*}{ Stride duration (s) } & PD & $1.10 \pm 0.09$ & $1.10 \pm 0.09$ & $1.12 \pm 0.08$ & \multirow[t]{2}{*}{ ns } & \multirow[t]{2}{*}{ UnW, Single $<$ Double } \\
\hline & Control & $1.07 \pm 0.10$ & $1.10 \pm 0.12$ & $1.13 \pm 0.11$ & & \\
\hline \multirow[t]{2}{*}{ Swing phase (\%) } & PD & $37.29 \pm 1.52$ & $38.32 \pm 2.00$ & $38.29 \pm 2.23$ & \multirow[t]{2}{*}{ ns } & \multirow[t]{2}{*}{ UnW $<$ Single, Double } \\
\hline & Control & $38.00 \pm 1.58$ & $38.60 \pm 1.65$ & $38.56 \pm 1.72$ & & \\
\hline \multirow[t]{2}{*}{ Stride velocity $(\mathrm{cm} / \mathrm{s})$} & PD & $103.77 \pm 16.31$ & $93.11 \pm 15.59$ & $92.24 \pm 14.91$ & \multirow[t]{2}{*}{$\mathrm{PD}<$ Control } & \multirow[t]{2}{*}{ UnW $<$ Single, Double } \\
\hline & Control & $114.64 \pm 17.73$ & $108.19 \pm 18.39$ & $107.06 \pm 17.17$ & & \\
\hline
\end{tabular}

ns: non significant. 
Table 3

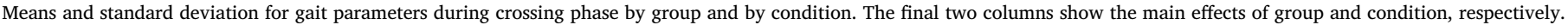

\begin{tabular}{|c|c|c|c|c|c|}
\hline Gait parameters & Group & Single & Double & Effects of group & Effects of condition \\
\hline \multicolumn{6}{|l|}{ Leading Limb } \\
\hline \multirow[t]{2}{*}{ Step length $(\mathrm{cm})$} & PD & $62.43 \pm 7.17$ & $60.85 \pm 5.48$ & ns & Single $>$ Double \\
\hline & Control & $68.61 \pm 9.33$ & $63.43 \pm 5.33$ & & \\
\hline \multirow[t]{2}{*}{ Step width $(\mathrm{cm})$} & PD & $11.52 \pm 4.02$ & $12.78 \pm 3.75$ & ns & ns \\
\hline & Control & $10.65 \pm 3.97$ & $10.09 \pm 3.03$ & & \\
\hline \multirow[t]{2}{*}{ Step duration (s) } & PD & $0.78 \pm 0.12$ & $0.77 \pm 0.09$ & ns & ns \\
\hline & Control & $0.73 \pm 0.08$ & $0.74 \pm 0.08$ & & \\
\hline \multirow[t]{2}{*}{ Swing phase (\%) } & $\mathrm{PD}$ & $83.28 \pm 2.02$ & $83.21 \pm 2.07$ & ns & ns \\
\hline & Control & $84.23 \pm 2.17$ & $83.85 \pm 2.38$ & & \\
\hline \multirow[t]{2}{*}{ Step velocity $(\mathrm{cm} / \mathrm{s})$} & $\mathrm{PD}$ & $82.09 \pm 15.83$ & $80.62 \pm 12.47$ & ns & Single $>$ Double \\
\hline & Control & $96.01 \pm 19.23$ & $86.93 \pm 13.26$ & & \\
\hline \multirow[t]{2}{*}{ Horizontal distance before obstacle $(\mathrm{cm})$} & $\mathrm{PD}$ & $74.75 \pm 8.91$ & $75.39 \pm 9.75$ & $\mathrm{PD}<$ Control & ns \\
\hline & Control & $86.11 \pm 16.9$ & $84.70 \pm 11.89$ & & \\
\hline \multirow[t]{2}{*}{ Horizontal distance after obstacle (cm) } & $\mathrm{PD}$ & $23.28 \pm 6.46$ & $21.49 \pm 3.98$ & ns & Single $>$ Double \\
\hline & Control & $26.15 \pm 5.94$ & $21.98 \pm 4.07$ & & \\
\hline \multirow[t]{2}{*}{ Toe clearance $(\mathrm{cm})$} & PD & $15.06 \pm 2.91$ & $14.47 \pm 3.32$ & $\mathrm{PD}<$ Control & ns \\
\hline & Control & $16.55 \pm 2.13$ & $16.56 \pm 2.21$ & & \\
\hline \multicolumn{6}{|l|}{ Trailing Limb } \\
\hline \multirow[t]{2}{*}{ Step length $(\mathrm{cm})$} & PD & $54.32 \pm 7.82$ & $49.21 \pm 4.32$ & ns & Single $>$ Double \\
\hline & Control & $61.76 \pm 9.64$ & $49.89 \pm 4.13$ & & \\
\hline \multirow[t]{2}{*}{ Step width (cm) } & $\mathrm{PD}$ & $13.42 \pm 4.41$ & $13.09 \pm 4.24$ & ns & ns \\
\hline & Control & $11.33 \pm 3.58$ & $11.09 \pm 3.09$ & & \\
\hline \multirow[t]{2}{*}{ Step duration (s) } & PD & $0.74 \pm 0.08$ & $0.75 \pm 0.11$ & ns & ns \\
\hline & Control & $0.68 \pm 0.08$ & $0.69 \pm 0.08$ & & \\
\hline \multirow[t]{2}{*}{ Swing phase (\%) } & $\mathrm{PD}$ & $78.71 \pm 2.68$ & $78.69 \pm 2.67$ & ns & Single $>$ Double \\
\hline & Control & $81.23 \pm 2.60$ & $79.91 \pm 2.60$ & & \\
\hline \multirow[t]{2}{*}{ Step velocity $(\mathrm{cm} / \mathrm{s})$} & $\mathrm{PD}$ & $74.41 \pm 15.74$ & $66.70 \pm 10.11$ & ns & Single $>$ Double \\
\hline & Control & $91.90 \pm 19.47$ & $72.83 \pm 9.59$ & & \\
\hline \multirow[t]{2}{*}{ Horizontal distance before obstacle $(\mathrm{cm})$} & $\mathrm{PD}$ & $23.44 \pm 4.53$ & $23.65 \pm 4.62$ & ns & ns \\
\hline & Control & $26.24 \pm 7.72$ & $25.26 \pm 4.25$ & & \\
\hline \multirow[t]{2}{*}{ Horizontal distance after obstacle (cm) } & $\mathrm{PD}$ & $93.71 \pm 13.04$ & $86.45 \pm 5.71$ & ns & Single $>$ Double \\
\hline & Control & $104.21 \pm 14.47$ & $87.68 \pm 4.93$ & & \\
\hline \multirow[t]{2}{*}{ Toe clearance $(\mathrm{cm})$} & $\mathrm{PD}$ & $23.25 \pm 5.41$ & $21.06 \pm 4.92$ & ns & Single $>$ Double \\
\hline & Control & $24.83 \pm 5.51$ & $22.33 \pm 3.40$ & & \\
\hline
\end{tabular}

ns: non significant.

be modulated by the basal ganglia [28]. Thus, due to dysfunctions in the basal ganglia in PD, the cognitive resources become overloaded and affect gait control more in people with PD than healthy individuals [29].

The main effect of condition in the crossing phase for leading and trailing limb demonstrated that the presence of the Second Double influenced the necessary adjustments to cross the First Double successfully. The increase in stride duration was necessary for the both PD and control groups to planning the obstacle crossing. Both groups made spatial and temporal adjustments (decreased of step length, velocity, swing phase and horizontal distance after obstacle) to step over the obstacle safely. Although our everyday environment are not adjusted proportionally to our size, the use of a single distance between obstacles led the healthy older people to perform larger adjustments due to the longer step length.

The second obstacle influenced the trailing toe clearance during step over the First Double. A reduction in the toe clearance could increase the likelihood of trip and fall [10]. This suggest that both groups prematurely transferred their attention to the second obstacle before completing the ongoing step, which is associated with loss of accuracy and precision of stepping movements and is likely to increase the incidence of trips and falls $[15,16]$. In addition, the PD group presented lower leading toe clearance and shorter horizontal distance before the obstacle compared to the control group. Previous studies have indicated that reduction in these distances increases the likelihood of tripping $[10,11]$. These results confirm deficits in movement amplitude regulation (hypokinesia) in people with PD, especially during complex task [11]. Thus, our results suggest that crossing one or two obstacles represents a risky task for people with PD.

Even with consistent and novel results, this study has a limitation.
Due to the fixed distance between obstacles, some results should be carefully analyzed, such as the decrease in variables during first double crossing. In this way, we suggest that future studies modulate the distance between obstacles to better understand gait adjustments in environments with multiple obstacles.

In conclusion, to deal with the increased sensorimotor and central processing demands of approaching to obstacles, people with PD adopted a more conservative locomotor behavior than healthy individuals. Both PD and control groups need more time to obtain and process environmental information and plan the action in environments with multiple obstacles. In addition, the Second Double influenced the trailing toe clearance during avoidance of the First Double in both groups, which could increase the incidence of trips and falls.

\section{Conflict of interest}

None.

\section{Acknowledgement}

The authors thank Sao Paulo Research Foundation (FAPESP) (grant number: \# 2013/27032-0) for financial support.

\section{References}

[1] H. Stolze, S. Klebe, C. Zechlin, C. Baecker, L. Friege, G. Deuschl, Falls in frequent neurological diseases-prevalence, risk factors and aetiology, J. Neurol. 251 (2004) 79-84.

[2] Q.J. Almeida, J.S. Frank, E.A. Roy, M.E. Jenkins, S. Spaulding, A.E. Patla, et al., An evaluation of sensorimotor integration during locomotion toward a target in Parkinson's disease, Neuroscience 134 (2005) 283-293.

[3] J. Konczak, D.M. Corcos, F. Horak, H. Poizner, M. Shapiro, P. Tuite, et al., 
Proprioception and motor control in Parkinson's disease, J. Mot. Behav. 41 (2009) $543-552$.

[4] F. Pieruccini-Faria, J.A. Jones, Q.J. Almeida, Motor planning in Parkinson's disease patients experiencing freezing of gait: the influence of cognitive load when approaching obstacles, Brain Cogn. 87 (2014) 76-85.

[5] F. Pieruccini-Faria, K.A. Ehgoetz Martens, C.R. Silveira, J.A. Jones, Q.J. Almeida, Interactions between cognitive and sensory load while planning and controlling complex gait adaptations in Parkinson's disease, BMC Neurol. 14 (2014) 250.

[6] S. O'Shea, M.E. Morris, R. Iansek, Dual task interference during gait in people with Parkinson disease: effects of motor versus cognitive secondary tasks, Phys. Ther. 82 (2002) 888-897.

[7] K.A. Martens, Q.J. Almeida, Dissociating between sensory and perceptual deficits in PD: more than simply a motor deficit, Mov. Disord. 27 (2012) 387-392.

[8] B. Galna, A.T. Murphy, M.E. Morris, Obstacle crossing in people with Parkinson's disease: foot clearance and spatiotemporal deficits, Hum. Mov. Sci. 29 (2010) 843-852.

[9] F. Pieruccini-Faria, R. Vitorio, Q.J. Almeida, C.R. Silveira, M.J. Caetano, F. Stella, et al., Evaluating the acute contributions of dopaminergic replacement to gait with obstacles in Parkinson's disease, J Mot. Behav. 45 (2013) 369-380.

[10] R. Vitorio, E. Lirani-Silva, A.M. Baptista, F.A. Barbieri, P.C. dos Santos, C. TeixeiraArroyo, et al., Disease severity affects obstacle crossing in people with Parkinson's disease, Gait Posture 40 (2014) 266-269.

[11] R. Vitorio, F. Pieruccini-Faria, F. Stella, S. Gobbi, L.T. Gobbi, Effects of obstacle height on obstacle crossing in mild Parkinson's disease, Gait Posture 31 (2010) 143-146.

[12] J.R. Berard, L.A. Vallis, Characteristics of single and double obstacle avoidance strategies: a comparison between adults and children, Exp. Brain Res. 175 (2006) 21-31.

[13] C.R. Lowrey, A. Watson, L.A. Vallis, Age-related changes in avoidance strategies when negotiating single and multiple obstacles, Exp. Brain Res. 182 (2007) 289-299.

[14] J. Krell, A.E. Patla, The influence of multiple obstacles in the travel path on avoidance strategy, Gait Posture 16 (2002) 15-19.

[15] R. Vitorio, L.T. Gobbi, E. Lirani-Silva, R. Moraes, Q.J. Almeida, Synchrony of gaze and stepping patterns in people with Parkinson's disease, Behav. Brain Res. 307 (2016) 159-164.

[16] G.J. Chapman, M.A. Hollands, Evidence that older adult fallers prioritise the planning of future stepping actions over the accurate execution of ongoing steps during complex locomotor tasks, Gait Posture 26 (2007) 59-67.
[17] F. Pieruccini-Faria, J.A. Jones, Q.J. Almeida, Insight into dopamine-dependent planning deficits in Parkinson's disease: a sharing of cognitive \& sensory resources, Neuroscience 318 (2016) 219-229.

[18] A.J. Hughes, S.E. Daniel, L. Kilford, A.J. Lees, Accuracy of clinical diagnosis of idiopathic Parkinson's disease: a clinico-pathological study of 100 cases, J. Neurol. Neurosurg. Psychiatry 55 (1992) 181-184.

[19] M.M. Hoehn, M.D. Yahr, Parkinsonism: onset, progression and mortality, Neurology 17 (1967) 427-442.

[20] C.L. Tomlinson, R. Stowe, S. Patel, C. Rick, R. Gray, C.E. Clarke, Systematic review of levodopa dose equivalency reporting in Parkinson's disease, Mov. Disord. 25 (2010) 2649-2653.

[21] R.J. Uitti, Y. Baba, Z.K. Wszolek, D.J. Putzke, Defining the Parkinson's disease phenotype: initial symptoms and baseline characteristics in a clinical cohort, Parkinsonism Relat. Disord. 11 (2005) 139-145.

[22] S.M. Brucki, R. Nitrini, P. Caramelli, P.H. Bertolucci, I.H. Okamoto, [Suggestions for utilization of the mini-mental state examination in Brazil], Arq. Neuropsiquiatr. 61 (2003) 777-781.

[23] L.S. Chou, K.R. Kaufman, M.E. Hahn, R.H. Brey, Medio-lateral motion of the center of mass during obstacle crossing distinguishes elderly individuals with imbalance, Gait Posture 18 (2003) 125-133.

[24] F.A. Barbieri, P.C. dos Santos, L. Simieli, D. Orcioli-Silva, J.H. van Dieen, L.T. Gobbi, Interactions of age and leg muscle fatigue on unobstructed walking and obstacle crossing, Gait Posture 39 (2014) 985-990.

[25] A.A. Mohagheghi, R. Moraes, A.E. Patla, The effects of distant and on-line visua information on the control of approach phase and step over an obstacle during locomotion, Exp. Brain Res. 155 (2004) 459-468.

[26] J. Haefeli, S. Vogeli, J. Michel, V. Dietz, Preparation and performance of obstacle steps: interaction between brain and spinal neuronal activity, Eur. J. Neurosci. 33 (2011) 338-348.

[27] D.S. Marigold, T. Drew, Contribution of cells in the posterior parietal cortex to the planning of visually guided locomotion in the cat: effects of temporary visual interruption, J. Neurophysiol. 105 (2011) 2457-2470.

[28] G.E. Alexander, M.D. Crutcher, M.R. DeLong, Basal ganglia-thalamocortical circuits: parallel substrates for motor, oculomotor, prefrontal and limbic functions, Prog. Brain Res. 85 (1990) 119-146.

[29] M. Desmurget, S.T. Grafton, P. Vindras, H. Grea, R.S. Turner, The basal ganglia network mediates the planning of movement amplitude, Eur. J. Neurosci. 19 (2004) 2871-2880. 